\title{
Cerebral sterile inflammation in neurodegenerative diseases
}

\author{
Kento Otani ${ }^{1,2}$ and Takashi Shichita ${ }^{1,3^{*}}$
}

\begin{abstract}
Therapeutic strategies for regulating neuroinflammation are expected in the development of novel therapeutic agents to prevent the progression of central nervous system (CNS) pathologies. An understanding of the detailed molecular and cellular mechanisms of neuroinflammation in each CNS disease is necessary for the development of therapeutics. Since the brain is a sterile organ, neuroinflammation in Alzheimer's disease (AD), Parkinson's disease $(\mathrm{PD})$, and amyotrophic lateral sclerosis (ALS) is triggered by cerebral cellular damage or the abnormal accumulation of inflammatogenic molecules in CNS tissue through the activation of innate and acquired immunity. Inflammation and CNS pathologies worsen each other through various cellular and molecular mechanisms, such as oxidative stress or the accumulation of inflammatogenic molecules induced in the damaged CNS tissue. In this review, we summarize the recent evidence regarding sterile immune responses in neurodegenerative diseases.
\end{abstract}

Keywords: Neuroinflammation, Alzheimer's disease, Parkinson's disease, Amyotrophic lateral sclerosis

\section{Background}

Inflammation is an important biological process in the pathologies of CNS. The cell death induced by various brain pathologies such as neurodegenerative diseases, ischemia, hemorrhage, trauma, and so on triggers inflammation. Conversely, inflammation in the peripheral organs or central nervous system can induce brain cell death. Some reports have demonstrated the relationships between the intestinal microbiome and cerebral inflammation. For example, gut microbiome dysbiosis is related to the dysregulation of the gut-brain axis and supports the neuroinflammatory responses in the brain, leading to the enhanced pathophysiology of AD [1]. Inflammation and brain pathologies thus worsen each other through various cellular and molecular mechanisms, leading to a poor prognosis in terms of quality of life.

The brain consists of various cells, including neurons, astrocytes, microglia, oligodendrocytes, and endothelial

\footnotetext{
* Correspondence: shichita-tk@igakuken.or.jp

'Stroke Renaissance Project, Tokyo Metropolitan Institute of Medical Science,

2-1-6 Kamikitazawa, Setagaya-ku, Tokyo 156-8506, Japan

${ }^{3}$ Precursory Research for Innovative Medical Care (PRIME), Japan Agency for

Medical Research and Development (AMED), Tokyo 100-0004, Japan

Full list of author information is available at the end of the article
}

cells. If cerebral pathologies exist, all of these kinds of cells are implicated in cerebral inflammation. Neurons, astrocytes, and oligodendrocytes are activated by pathological insults and can become inflammatogenic factors. For example, oxidative stress within brain cells causes neurodegeneration in $\mathrm{AD}, \mathrm{PD}$, and ALS [2]. Brain trauma or ischemia induces oxidative stress in brain cells [3]. These oxidative stresses induce the production of reactive oxygen species (ROS) from damaged brain cells, which enhance the vascular dysfunction and the infiltration of circulating immune cells [4]. ROS loosen the cerebral vasculature by decreasing the tight junction proteins and activating matrix metalloproteinases (MMPs) in endothelial cells, resulting in the disruption of the blood-brain barrier (BBB) which causes the infiltration of circulating immune cells and inflammatory factors into the brain [5]. Oxidative stress also induces the production of inflammatory cytokines in macrophages [6].

The cell death of brain cells triggers inflammation. Brain trauma and ischemia directly injures brain tissue and induces the necrotic cell death of various brain cells. Neurodegeneration-related inflammation and cellular stresses also cause neuronal and glial cell death. This

(c) The Author(s). 2020 Open Access This article is licensed under a Creative Commons Attribution 4.0 International License, which permits use, sharing, adaptation, distribution and reproduction in any medium or format, as long as you give

appropriate credit to the original author(s) and the source, provide a link to the Creative Commons licence, and indicate if changes were made. The images or other third party material in this article are included in the article's Creative Commons licence, unless indicated otherwise in a credit line to the material. If material is not included in the article's Creative Commons licence and your intended use is not permitted by statutory regulation or exceeds the permitted use, you will need to obtain permission directly from the copyright holder. To view a copy of this licence, visit http://creativecommons.org/licenses/by/4.0/ 
releases intracellular components into the extracellular space that triggers inflammation by activating immune cells $[7,8]$. Uric acid and adenosine triphosphate (ATP) released from injured mammalian cells trigger the maturation of dendritic cells and subsequent immune responses of CD4- or CD8-positive T cells [9, 10]. ATP is well known as a "find me" signal that is released from injured neurons and astrocytes. Extracellular ATP induces chemotaxis and the production of inflammatory cytokines and ROS in microglia through the activation of GTP-binding protein-coupled P2 receptors [11, 12]. Among various P2 receptors, P2Y12 receptors were recently shown to play pivotal roles in neuroprotection and the maintenance of neuronal activities by adjacent microglia [13]. Extracellular ATP release from astrocytes also regulates the susceptibility to chronic social defeat and modulates depressive-like behaviors, indicating the astrocytic ATP release-dependent regulation of neuronal function against social stresses [14]. Thus, ATP functions as a key molecule for not only the maintenance of neural function but also as the trigger of inflammation in pathologic brain conditions, which may be regulated by extracellular levels of ATP released from brain cells or the expression levels of P2 receptors in microglia. ATP also modifies the function of endothelial cells and myeloid cells other than microglia. The extracellular release of ATP induces the expression of MMP9 and IL$1 \beta$ in injured brain tissue [15]. These inflammatory molecules promote the disruption of $\mathrm{BBB}$ through the degradation of tight junction proteins and endothelial cell death [16]. BBB disruption promotes the infiltration of circulating immune cells in the injured brain. ATP also triggers inflammation by these infiltrating immune cells. Importantly, ATP is an activator of the inflammasome complex that induces the production of $\mathrm{IL}-1 \beta$ and IL-18 via caspase- 1 activation and pyroptotic cell death [17]. IL-1 $\beta$ contributes to neuronal cell death by increasing the entry of calcium ion through N-methyl-D-aspartate (NMDA) receptor [18]. Inflammasome activation has been reported to be implicated in the pathologies of various CNS diseases, including $\mathrm{AD}, \mathrm{PD}, \mathrm{ALS}$, and stroke [19-22]. Molecules associated with neurodegeneration and demyelination, such as lysophosphatidylcholine, also activate the inflammasome [23]. Although inflammasome activation occurs in both infiltrating immune cells and neurons and other glial cells [24], the establishment of the inflammatory environment generated by inflammasome activation and the subsequent pyroptotic cell death exaggerates the neuronal dysfunction and neurodegenerative CNS pathologies [25].

In addition to the extracellular release of ATP, some endogenous-specific molecules released from damaged brain cells trigger inflammation. These molecules, called damage-associated molecular patterns (DAMPs), transmit the damage signal to brain cells around the lesion and induce responses for the adaptation to pathological conditions. DAMPs activate immunological receptors in cerebral myeloid cells and other brain cells to produce inflammatory factors. Microglia are the resident cerebral myeloid cells derived from the yolk sac that infiltrate the brain around embryonic day 7 [26]. Microglia and infiltrating macrophages and neutrophils are major producers of inflammatory cytokines. Pattern recognition receptors (PRRs) are important immunological receptors for the recognition of DAMPs released from damaged brain cells. Toll-like receptors (TLRs), receptors for advanced glycation end products (RAGE), and macrophage-inducible Ctype lectin (Mincle) are implicated in the triggering or promotion of cerebral inflammation. For example, the accumulation of amyloid $\beta$ or $\alpha$-synuclein is thought to cause neurodegeneration in Alzheimer's disease and Parkinson's disease, respectively. Amyloid $\beta$ peptide $(A \beta)$ and $\alpha$-synuclein are recognized by TLRs to induce neuroinflammation, which promotes glial scar formation and neurodegeneration [27, 28]. The activation of TLR4 signaling in the neuron which is likely responsible for the accumulation of $A \beta$ increases the further expression of $A \beta$ in the neuron and results in the progression of $A D$ [29]. Traumatic or ischemic brain injury induces the extracellular release of DAMPs from damaged brain cells. Mitochondrial DNA and formylated peptides, high mobility group box 1 (HMGB1), and peroxiredoxin family proteins (PRXs) are DAMPs that trigger neuroinflammation through the activation of TLRs and RAGE in cerebral myeloid cells [30, 31]. These inflammatogenic pathways trigger the production of inflammatory cytokines, such as IL-12 and IL-23, which induce the subsequent chronic inflammation mediated by lymphocytes.

Cerebral inflammation worsens CNS pathologies and causes further generation of inflammatogenic molecules and extracellular DAMPs from damaged brain cells. It is possible that chronic neurodegenerative diseases are caused by this vicious cycle between inflammation and brain damage. Recent studies have clarified the importance of immune responses and immunological receptors in the clearance of inflammatogenic molecules and extracellular DAMPs from the damaged brain. For example, the immune checkpoint blockade against programmed death-1 (PD-1) promotes the clearance of $\mathrm{A} \beta$ through the enhanced interferon (IFN)- $\gamma$-mediated systemic immune responses in the murine model of Alzheimer's disease [32]. Currently, therapeutic strategies for regulating neuroinflammation are expected in the development of novel therapeutic agents to prevent CNS pathologies. An understanding of the detailed molecular and cellular mechanisms of neuroinflammation in each CNS disease is necessary for the development of therapeutics (Fig. 1). 


\section{Alzheimer's disease}

$\mathrm{AD}$ is the most frequent cause of dementia, which is characterized by progressive cognitive impairment. The temporal and parietal lobes, the hippocampal region in particular, become markedly atrophic in patients with ADs. This is due to the loss of neurons and synapses as a result of progressive neurodegeneration. The accumulation of $\mathrm{A} \beta$ and the deposition of neurofibrillary tangles composed of aggregated tau protein have been considered the major pathogenic cause of $\mathrm{AD}$ (the amyloid cascade hypothesis) although the cause of these histopathologies has not yet been clarified. Inflammation is also implicated in the pathogenic process of $\mathrm{AD}$, given that the extracellular deposits of $\mathrm{A} \beta$ trigger neuroinflammation, and this inflammatory response can promote the intracellular aggregation of tau within vulnerable neurons [33]. Microglia are the primary cells that drive neuroinflammation and gliosis around $A \beta$ plaques with reactive astrocytes $[34,35]$. Some of the immunological receptors that interact with $A \beta$ are expected to be important to the inflammatory responses and disease progression in $\mathrm{AD}$ pathologies. Indeed, recent studies have identified several receptors in microglia that are strongly related to neuroinflammation in AD. TLR4, RAGE, CD36, and triggering receptor expressed on myeloid cells 2 (TREM2) are receptors for $A \beta$ and induce the production of inflammatory cytokines, complement components, and chemokines [36-38]. Microglia also function as phagocytic cells that remove pathogenic molecules and aggregated proteins, indicating that

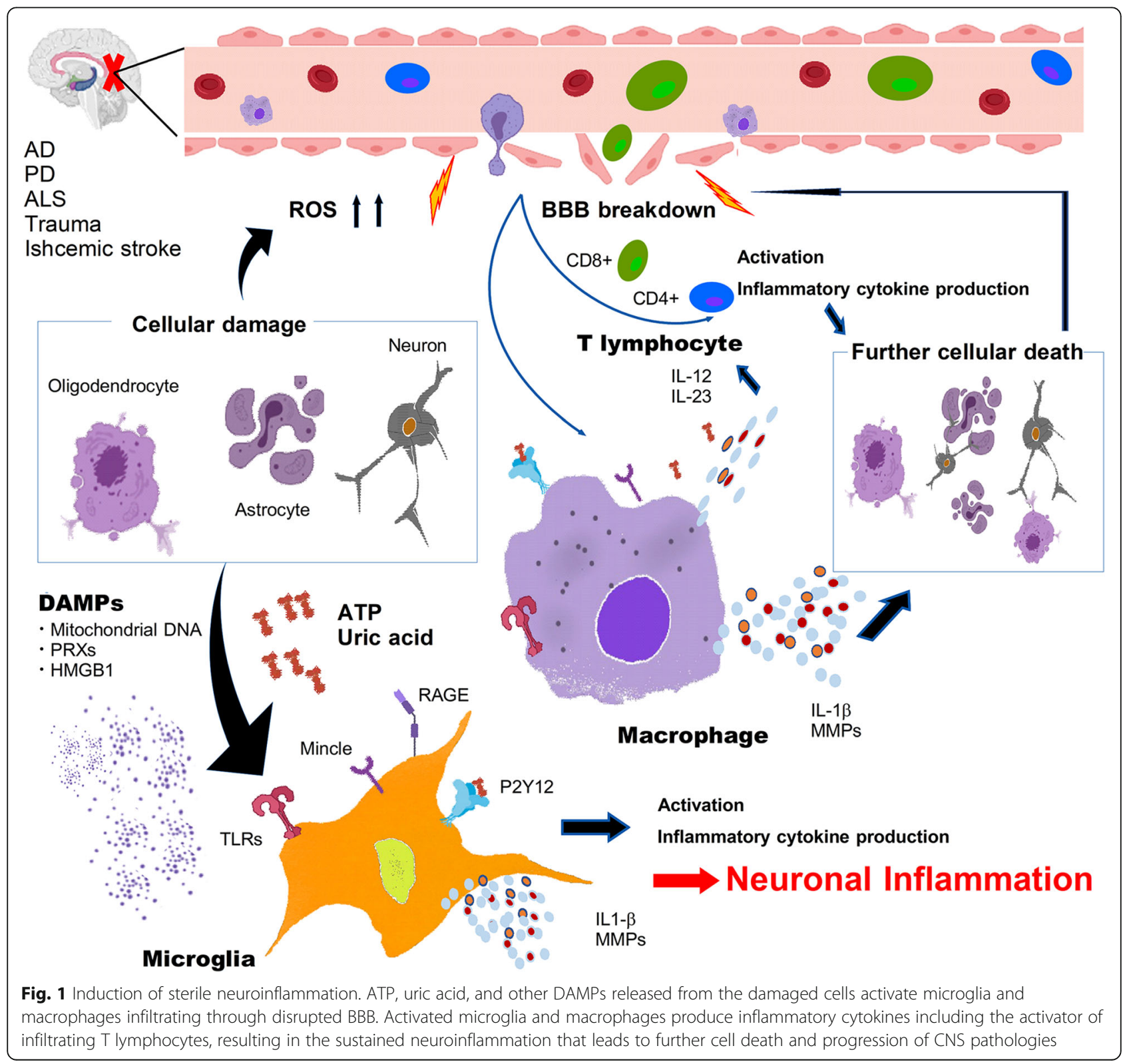


microglia-related inflammation and the removal of inflammatogenic molecules may be the keys to understanding the $\mathrm{AD}$ pathologies.

The normal function of $\mathrm{BBB}$ is important for the cerebral accumulation of $A \beta$, since $B B B$ controls $A \beta$ entry from plasma and $A \beta$ clearance from the brain [39]. $A \beta$ is recognized by RAGE expressed in endothelial cells, and this RAGE- $\mathrm{A} \beta$ interaction disrupts $\mathrm{BBB}$ by promoting the degradation of tight junction proteins and the secretion of MMPs [40]. Although BBB disruption is implicated in the pathogenesis of $\mathrm{AD}, \mathrm{A} \beta$-mediated RAGE activation in microglia also contributes to the vulnerability of endothelial cells and synaptic dysfunction [41, 42]. Thus, RAGE is a multiligand receptor, which was initially identified as a receptor for advanced glycation end products (AGEs) for the induction of neuroinflammation in $\mathrm{AD}$, leading to accelerated neuronal damage and BBB dysfunction [43].

CD36, which is a class $B$ scavenger receptor that is expressed in microglia and endothelial cells, is also a receptor for fibrillar $\mathrm{A} \beta[44,45]$. Inflammatory responses in microglia stimulated by fibrillar $A \beta$ are largely dependent on CD36, given that CD36-deficient microglia produce much fewer inflammatory cytokines, chemokines, and ROS than microglia from wild-type mice do when microglia are treated with fibrillar $A \beta$ [46]. A $\beta$-mediated CD36 activation was also reported to generate the senescence-associated secretory phenotype (SASP) [47]. These studies revealed that CD36 bound to $\mathrm{A} \beta$ induces the recruitment of microglia and the production of inflammatory mediators in the AD brain.

Owing to recent genome-wide association studies (GWASs), TREM2 has been identified as a receptor associated with a high risk of developing AD; a substitution mutation, the $\mathrm{R} 47 \mathrm{H}$ variant of TREM2, is highly associated with AD $[48,49]$. TREM2, which is expressed by microglia and neurons in the brain, is important for triggering phagocytosis and inflammatory responses [50, 51]. $\mathrm{R} 47 \mathrm{H}$ mutation of TREM 2 impairs microglial activation and the microgliosis around $\mathrm{A} \beta$ plaque [52, 53]. TREM 2 binds directly to $A \beta$ oligomer and plays a pivotal role in the degradation of $A \beta$ through the proteasome pathway within microglia $[54,55]$. Singlecell transcriptional profiling of microglia associated with $\mathrm{AD}$ revealed the important role of TREM2 in the upregulation of genes related to phagocytosis and lipid metabolism [56]. The possibility of TREM2 actions downstream of CD33, another microglial receptor associated with a high risk of $\mathrm{AD}$, has been demonstrated, which regulates IL- $1 \beta$-mediated inflammatory cascades in $\mathrm{AD}$ pathologies [57].

\section{Parkinson's disease}

$\mathrm{PD}$ is a slowly progressive neurodegenerative disease that mainly impairs the motor system controlling muscle tonus. This is due to the loss of dopaminergic neurons in the substantia nigra. Lewy bodies observed in brain lesion sites are the defining characteristics of PD and are deposits made of a filament structure containing $\alpha$ synuclein [58]. The abnormal accumulation of $\alpha$ synuclein in the brain, which is caused by Snca gene polymorphisms, dysfunction of a protein degradation system such as proteases, autophagy, or the ubiquitinproteasome system, is considered to be the major cause of PD pathologies [59-61]. The aggregation of $\alpha$ synuclein is dependent on a seeding-nucleation mechanism that leads to fibril growth [62]. Although the intracellular aggregation of $\alpha$-synuclein protein is the pathological characteristic of PD, $\alpha$-synuclein pathologies are considered to propagate from neuron to neuron, as in prion disease [63].

Extracellular $\alpha$-synuclein is an inflammatogenic molecule that contributes to the progression of neuroinflammation and pathologies in PD $[64,65]$. Fibrillar $\alpha$ synuclein activates TLR2 and the nucleotide oligomerization domain-like receptor pyrin domain containing 3 (NLRP3) inflammasome to produce IL-1 $\beta$, which is a major cytokine implicated in the initiation and progression of PD [66-69]. Increased expression levels of IL-1 $\beta$ and NLRP3 are observed in the serum of patients with PD [70]. Nitrated $\alpha$-synuclein triggers both microglia-mediated neuroinflammation and lymphocyte-mediated acquired immune responses that worsen PD pathologies [71, 72]. Microglial TLR4 is also activated by soluble or fibrillar $\alpha$-synuclein to induce the production of inflammatory cytokines, chemokines, and ROS [73]. Among chemokines, CXCL12 is important for the accumulation of microglia activated by $\alpha$-synuclein [74].

PTEN-induced putative kinase 1 (PINK1) and PARK IN have been identified as causal genes associated with familiar PD [75, 76]. PINK1, a serine/threonine kinase, generates phosphorylated ubiquitin, which induces the full activation of the ubiquitin ligase activity of PARKIN [77]. PINK1 and PARKIN are important for the removal of damaged mitochondria through mitophagy and the prevention of neuroinflammation, since mitochondrial damage causes the extracellular release of mitochondrial DAMPs [78]. Mitochondrial DNA stress induces the activation of the cGAS-STING pathway [79]; in fact, a deficiency of STING recovers the loss of dopaminergic neurons in the substantia nigra of aged Parkin-deficient mice. Although the relationships between mitochondrial dysfunction and $\alpha$-synuclein accumulation remain to be clarified, two recent reports suggest the important roles of $\alpha$-synuclein in the regulation of mitochondrial function $[80,81]$. Thus, $\alpha$-synuclein-mediated neuroinflammation is essential for the generation of $\mathrm{PD}$ pathologies. 


\section{Amyotrophic lateral sclerosis}

ALS is a slowly progressive neurodegenerative disease. Neurodegeneration in ALS impairs motor neuron function in the CNS [82]. The accumulation of rounded or thread-like deposits containing TAR DNA binding protein 43 (TDP-43) is considered to play a pivotal role in the progression of ALS pathologies [83]. The inclusion body generated by the accumulation of abnormally phosphorylated TDP-43 is important to the progression of ALS [84, 85]. Recently, shortened TDP43 splice variants have been demonstrated to preferentially accumulate within neurons and exert a neurotoxic effect, leading to progressive neurodegeneration in ALS [86]. The extracellular TDP-43 activates microglia through the CD14 receptor and enhances proinflammatory cascades such as NF- $\mathrm{kB}$ and AP-1 pathways, which are involved in the neurotoxicity of motor neurons [87].

Many studies have demonstrated that neuroinflammation induced by activated microglia and $\mathrm{T}$ lymphocytes is implicated in the progressive pathologies of ALS [88]. Microglia and astrocytes play an important role in ALS pathogenesis, given that cellular damage induced by mutant superoxide dismutase (SOD1) in microglia and astrocytes exaggerates the ALS pathologies [89, 90]. The accumulation of misfolded SOD1 induces inflammasome activation and IL-1 $\beta$ production, which is implicated in the progression of ALS pathologies [91]. The progressive and spreading motor neuron pathologies in ALS seem to be due to the propagation of misfolded proteins such as SOD1 [92]. The intracellular aggregation of misfolded SOD1 induces the production of inflammatory cytokines in microglia [93]. The administration of antibodies against misfolded SOD1 attenuates the ALS pathologies, indicating that the aggregation of misfolded SOD1 is one of the pivotal mechanisms causing motor neuron degeneration [94].

The infiltration of helper (CD4+) and cytotoxic (CD8+ ) $\mathrm{T}$ lymphocytes is observed in the neurodegenerative lesions of ALS [95]. IFN- $\gamma$-producing helper T (Th1) and IL-17-producing helper T (Th17) lymphocytes are inflammatory subtypes detected in the blood and cerebrospinal fluid of ALS [96, 97]. The deficiency in T

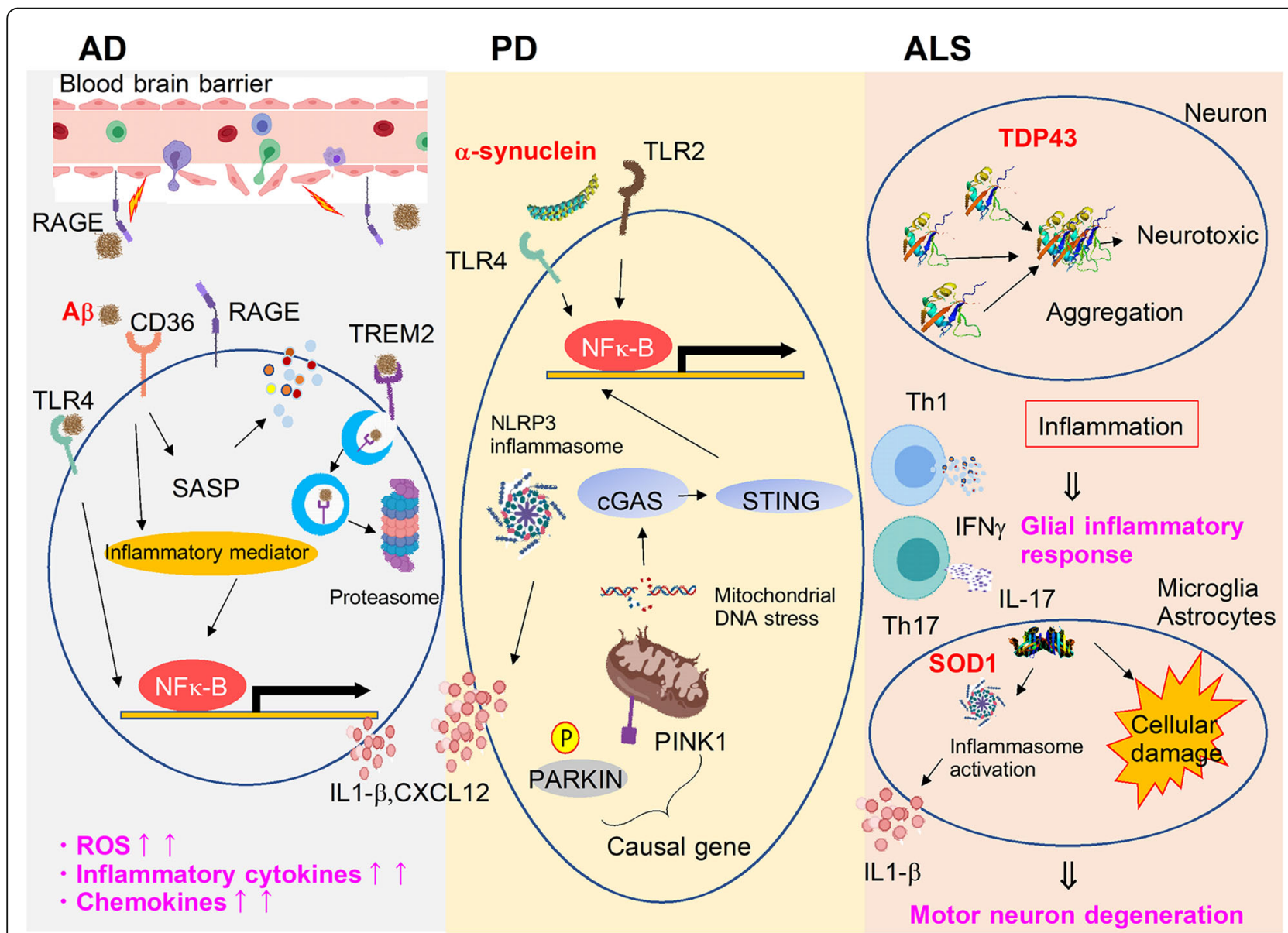

Fig. 2 Different mechanisms of neuroinflammation among AD, PD, and ALS. This is the summarized figure of molecular and cellular mechanisms of sterile neuroinflammation in AD, PD, and ALS. Different cells and molecules regulate the neuroinflammation that has a pivotal role in the induction of each CNS pathology 
lymphocyte function in the ALS model enhances the protection of motor neurons and attenuates the glial activation, however, suggesting the neuroprotective role of $\mathrm{T}$ lymphocytes in ALS pathologies [88, 98]. IL-4expressing regulatory $\mathrm{T}$ cells attenuate the activation of inflammatory microglial cells and prevent the progression of ALS pathologies [99]. Thus, the accumulation of inflammatogenic factors induces the activation of glial cells. T lymphocyte-mediated regulation of glial inflammatory responses is pivotal for the progression of ALS pathologies.

\section{Conclusion}

Neuroinflammation plays an important role in the progression of neurodegenerative diseases. The generation of inflammatogenic molecules due to the cellular damage or pathologies of neurodegenerative diseases induces inflammation by glial cells and immune cells. The clearance of these inflammatogenic molecules from brain lesions is also a result of the function of the glial or immune cells implicated in neuroinflammation. Thus, the immune-neural network in the pathologies of neurodegenerative diseases is complicated (Fig. 2). Finding the pathway that removes proinflammatory factors or promotes neural repair without causing inflammation will be necessary for developing therapeutic methods to regulate the inflammation in the pathophysiology of neurodegenerative diseases. Identification of the key molecules that regulate the inflammation and neural repair to improve CNS pathologies is important to the development of therapeutic methods for CNS diseases.

\begin{abstract}
Abbreviations
CNS: Central nervous system; AD: Alzheimer's disease; PD: Parkinson's disease ALS: Amyotrophic lateral sclerosis; ROS: Reactive oxygen species; MMPs: Matrix metalloproteinases; BBB: Blood-brain barrier; ATP: Adenosine triphosphate; DAMPs: Damage-associated molecular patterns; PRRs: Pattern recognition receptors; TLRs: Toll-like receptors; RAGE: Receptors for advanced glycation end products; Mincle: Macrophage-inducible C-type lectin; HMGB1: High mobility group box 1; PRXs: Peroxiredoxin family proteins; Aß: Amyloid beta; TREM2: Triggering receptor expressed on myeloid cells 2; AGEs: Advanced glycation end products; SASP: Senescence-associated secretory phenotype; GWASs: Genome-wide association studies; NLRP3: Nucleotide oligomerization domain-like receptor pyrin domain containing 3; PINK1: PTEN-induced putative kinase 1; TDP43: TAR DNA binding protein 43; SOD1: Superoxide dismutase
\end{abstract}

\section{Acknowledgements}

Not applicable.

\section{Authors' contributions}

T.O. and T.S wrote the manuscript. The authors read and approved the final manuscript.

\section{Funding}

This work was supported by PRIME from AMED under grant number JP18gm5910023 (T.S.); a Grant-in-Aid for Scientific Research on Innovative Areas (Dynamic Regulation of Brain Function by Scrap \& Build System) (19H04765) from the Ministry of Education, Culture, Sports, Science and Technology of Japan (MEXT) (T.S.); JSPS KAKENHI Grants-in-Aid for Young Scientists (17H05096) (T.S.); a Toray Science and Technology Grant (T.S.); and grants from the Mitsubishi Foundation (T.S.), the Naito Foundation (T.S.), the MSD Life Science Foundation (T.S.), the Senri Life Science Foundation (T.S.), and the Ono Medical Research Foundation (T.S.).

\section{Availability of data and materials}

Not applicable.

\section{Ethics approval and consent to participate}

As this is a literature review, and this study did not involve any intervention in humans, there is no need for ethics approval or consent to participate in this study.

\section{Consent for publication}

Not applicable.

\section{Competing interests}

The authors declare that they have no competing interests.

\section{Author details}

${ }^{1}$ Stroke Renaissance Project, Tokyo Metropolitan Institute of Medical Science, 2-1-6 Kamikitazawa, Setagaya-ku, Tokyo 156-8506, Japan. ²Division of Biochemistry, Faculty of Pharmacy and Graduate School of Pharmaceutical Science, Keio University, Tokyo 105-8512, Japan. ${ }^{3}$ Precursory Research for Innovative Medical Care (PRIME), Japan Agency for Medical Research and Development (AMED), Tokyo 100-0004, Japan.

Received: 14 May 2020 Accepted: 7 August 2020

Published online: 08 December 2020

\section{References}

1. Sochocka $M$, et al. The gut microbiome alterations and inflammation-driven pathogenesis of Alzheimer's disease-a critical review. Mol Neurobiol. 2019; 56:1841-51.

2. Niedzielska $\mathrm{E}$, et al. Oxidative stress in neurodegenerative diseases. Mol Neurobiol. 2016:53:4094-125.

3. Fraser PA. The role of free radical generation in increasing cerebrovascular permeability. Free Radic Biol Med. 2011;51:967-77.

4. Abdul-Muneer PM, Chandra N, Haorah J. Interactions of oxidative stress and neurovascular inflammation in the pathogenesis of traumatic brain injury. Mol Neurobiol. 2015;51:966-79.

5. Abdul-Muneer PM, et al. Induction of oxidative and nitrosative damage leads to cerebrovascular inflammation in an animal model of mild traumatic brain injury induced by primary blast. Free Radic Biol Med. 2013;60:282-91.

6. Hwang I, et al. Peroxiredoxin 3 deficiency accelerates chronic kidney injury in mice through interactions between macrophages and tubular epithelial cells. Free Radic Biol Med. 2019:131:162-72.

7. Kono H, Rock KL. How dying cells alert the immune system to danger. Nat Rev Immunol. 2008:8:279-89.

8. Chen GY, Nuñez G. Sterile inflammation: sensing and reacting to damage. Nat Rev Immunol. 2010;10:826-37.

9. Shi Y, Evans JE, Rock KL. Molecular identification of a danger signal that alerts the immune system to dying cells. Nature. 2003;425:516-21.

10. Idzko $M$, et al. Extracellular ATP triggers and maintains asthmatic airway inflammation by activating dendritic cells. Nat Med. 2007;13:913-9.

11. Inoue K. Microglial activation by purines and pyrimidines. Glia. 2002;40:15663.

12. Hu Y, et al. mTOR-mediated metabolic reprogramming shapes distinct microglia functions in response to lipopolysaccharide and ATP. Glia. 2019.

13. Cserép $\mathrm{C}$, et al. Microglia monitor and protect neuronal function via specialized somatic purinergic junctions: Science; 2019.

14. Cao X, et al. Astrocyte-derived ATP modulates depressive-like behaviors. Nat Med. 2013;19:773-7.

15. Yang F, Zhao K, Zhang $X$, Zhang J, Xu B. ATP Induces disruption of tight junction proteins via IL-1 beta-dependent MMP-9 activation of human blood-brain barrier. Neural Plast. 2016;2016:8928530

16. Subauste CS. The CD40-ATP-P2X. Front Immunol. 2019:10:2958.

17. Suzuki T, et al. Extracellular ADP augments microglial inflammasome and NF-kB activation via the P2Y12 receptor. Eur J Immunol. 2019.

18. Viviani $B$, et al. Interleukin-1 beta enhances NMDA receptor-mediated intracellular calcium increase through activation of the Src family of kinases. J Neurosci. 2003;23:8692-700. 
19. Ising $C$, et al. NLRP3 inflammasome activation drives tau pathology. Nature. 2019;575:669-73.

20. Gordon R, et al. Inflammasome inhibition prevents a-synuclein pathology and dopaminergic neurodegeneration in mice. Sci Transl Med. 2018;10.

21. Deora $V$, et al. The microglial NLRP3 inflammasome is activated by amyotrophic lateral sclerosis proteins. Glia. 2020;68:407-21.

22. Deroide $N$, et al. MFGE8 inhibits inflammasome-induced IL-1 $\beta$ production and limits postischemic cerebral injury. J Clin Invest. 2013;123:1176-81.

23. Freeman L, et al. NLR members NLRC4 and NLRP3 mediate sterile inflammasome activation in microglia and astrocytes. J Exp Med. 2017;214 1351-70.

24. Kaushal V, et al. Neuronal NLRP1 inflammasome activation of Caspase-1 coordinately regulates inflammatory interleukin-1-beta production and axonal degeneration-associated Caspase-6 activation. Cell Death Differ. 2015;22:1676-86.

25. Voet S, Srinivasan S, Lamkanfi M, van Loo G. Inflammasomes in neuroinflammatory and neurodegenerative diseases. EMBO Mol Med. 2019;11.

26. Ginhoux F, et al. Fate mapping analysis reveals that adult microglia derive from primitive macrophages. Science. 2010;330:841-5.

27. McDonald $\mathrm{CL}$, et al. Inhibiting TLR2 activation attenuates amyloid accumulation and glial activation in a mouse model of Alzheimer's disease. Brain Behav Immun. 2016;58:191-200.

28. Kouli A, Horne CB, Williams-Gray $\mathrm{CH}$. Toll-like receptors and their therapeutic potential in Parkinson's disease and a-synucleinopathies. Brain Behav Immun. 2019;81:41-51.

29. Wu D, Zhang $X$, Zhao M, Zhou AL. The role of the TLR4/NF-KB signaling pathway in $A \beta$ accumulation in primary hippocampal neurons. Sheng $L i$ Xue Bao. 2015:67:319-28.

30. Gülke E, Gelderblom M, Magnus T. Danger signals in stroke and their role on microglia activation after ischemia. Ther Adv Neurol Disord. 2018;11: 1756286418774254

31. B. Relja, W. G. Land, Damage-associated molecular patterns in trauma. Eur J Trauma Emerg Surg, (2019)

32. Baruch $\mathrm{K}$, et al. PD-1 immune checkpoint blockade reduces pathology and improves memory in mouse models of Alzheimer's disease. Nat Med. 2016; 22:135-7.

33. McGeer PL, McGeer EG. The amyloid cascade-inflammatory hypothesis of Alzheimer disease: implications for therapy. Acta Neuropathol. 2013;126: 479-97.

34. Heppner FL, Ransohoff RM, Becher B. Immune attack: the role of inflammation in Alzheimer disease. Nat Rev Neurosci. 2015;16:358-72.

35. Prokop S, Miller KR, Heppner FL. Microglia actions in Alzheimer's disease. Acta Neuropathol. 2013;126:461-77.

36. Cai Z, Hussain MD, Yan LJ. Microglia, neuroinflammation, and beta-amyloid protein in Alzheimer's disease. Int J Neurosci. 2014;124:307-21.

37. Lee JJ, Wang PW, Yang $\mathrm{H}, \mathrm{Wu} \mathrm{CL}$, Chuang JH. Amyloid-beta mediates the receptor of advanced glycation end product-induced pro-inflammatory response via toll-like receptor 4 signaling pathway in retinal ganglion cell line RGC-5. Int J Biochem Cell Biol. 2015;64:1-10.

38. Yu Y, Ye RD. Microglial A $\beta$ receptors in Alzheimer's disease. Cell Mol Neurobiol. 2015;35:71-83.

39. Shibata $M$, et al. Clearance of Alzheimer's amyloid-ss(1-40) peptide from brain by LDL receptor-related protein-1 at the blood-brain barrier. J Clin Invest. 2000;106:1489-99.

40. S. Y. Kook et al., $A \beta_{1-42}-R A G E$ interaction disrupts tight junctions of the blood-brain barrier via $\mathrm{Ca}^{2+}$-calcineurin signaling. J Neurosci 32, 8845-8854 (2012).

41. Criscuolo C, et al. Entorhinal Cortex dysfunction can be rescued by inhibition of microglial RAGE in an Alzheimer's disease mouse model. Sci Rep. 2017;7:42370

42. Origlia N, et al. Microglial receptor for advanced glycation end productdependent signal pathway drives beta-amyloid-induced synaptic depression and long-term depression impairment in entorhinal cortex. J Neurosci. 2010; 30:11414-25.

43. Wan $W$, Chen $H$, Li Y. The potential mechanisms of Aß-receptor for advanced glycation end-products interaction disrupting tight junctions of the blood-brain barrier in Alzheimer's disease. Int J Neurosci. 2014; 124:75-81.

44. Coraci IS, et al. CD36, a class B scavenger receptor, is expressed on microglia in Alzheimer's disease brains and can mediate production of reactive oxygen species in response to beta-amyloid fibrils. Am J Pathol. 2002;160:101-12.

45. Moore KJ, et al. A CD36-initiated signaling cascade mediates inflammatory effects of beta-amyloid. J Biol Chem. 2002;277:47373-9.

46. El Khoury JB, et al. CD36 mediates the innate host response to betaamyloid. J Exp Med. 2003:197:1657-66.

47. Chong $M$, et al. CD36 initiates the secretory phenotype during the establishment of cellular senescence. EMBO Rep. 2018;19.

48. Guerreiro R, et al. TREM2 variants in Alzheimer's disease. N Engl J Med. 2013; 368:117-27.

49. Jonsson T, Stefansson K. TREM2 and neurodegenerative disease. N Engl J Med. 2013;369:1568-9.

50. Takahashi K, Rochford CD, Neumann H. Clearance of apoptotic neurons without inflammation by microglial triggering receptor expressed on myeloid cells-2. J Exp Med. 2005;201:647-57.

51. Hsieh $\mathrm{CL}$, et al. A role for TREM2 ligands in the phagocytosis of apoptotic neuronal cells by microglia. J Neurochem. 2009;109:1144-56.

52. Song WM, et al. Humanized TREM2 mice reveal microglia-intrinsic and -extrinsic effects of R47H polymorphism. J Exp Med. 2018;215:745-60.

53. Wang $Y$, et al. TREM2 lipid sensing sustains the microglial response in an Alzheimer's disease model. Cell. 2015;160:1061-71.

54. Y. Zhao et al., TREM2 Is a receptor for $\beta$-amyloid that mediates microglial function. Neuron 97, 1023-1031.e1027 (2018).

55. Jay $T R$, et al. Disease progression-dependent effects of TREM2 deficiency in a mouse model of Alzheimer's disease. J Neurosci. 2017:37:637-47.

56. H. Keren-Shaul et al., A unique microglia type associated with restricting development of Alzheimer's disease. Cell 169, 1276-1290.e1217 (2017).

57. A. Griciuc et al., TREM2 acts downstream of CD33 in modulating microglial pathology in Alzheimer's disease. Neuron 103, 820-835.e827 (2019).

58. M. G. Spillantini, R. A. Crowther, R. Jakes, M. Hasegawa, M. Goedert, alphaSynuclein in filamentous inclusions of Lewy bodies from Parkinson's disease and dementia with Lewy bodies. Proc Natl Acad Sci U S A 95, 6469-6473 (1998).

59. Lashuel HA, Overk CR, Oueslati A, Masliah E. The many faces of a-synuclein: from structure and toxicity to therapeutic target. Nat Rev Neurosci. 2013;14: $38-48$.

60. Conway KA, Harper JD, Lansbury PT. Accelerated in vitro fibril formation by a mutant alpha-synuclein linked to early-onset Parkinson disease. Nat Med. 1998:4:1318-20

61. Webb JL, Ravikumar B, Atkins J, Skepper JN, Rubinsztein DC. Alpha-synuclein is degraded by both autophagy and the proteasome. J Biol Chem. 2003; 278:25009-13.

62. S. J. Wood et al., alpha-synuclein fibrillogenesis is nucleation-dependent. Implications for the pathogenesis of Parkinson's disease. J Biol Chem 274, 19509-19512 (1999).

63. Brundin P, Li JY, Holton JL, Lindvall O, Revesz T. Research in motion: the enigma of Parkinson's disease pathology spread. Nat Rev Neurosci. 2008;9 $741-5$.

64. Lee EJ, et al. Alpha-synuclein activates microglia by inducing the expressions of matrix metalloproteinases and the subsequent activation of protease-activated receptor-1. J Immunol. 2010;185:615-23.

65. Lema Tomé CM, et al. Inflammation and a-synuclein's prion-like behavior in Parkinson's disease--is there a link? Mol Neurobiol. 2013:47:561-74.

66. Kim C, et al. Neuron-released oligomeric a-synuclein is an endogenous agonist of TLR2 for paracrine activation of microglia. Nat Commun. 2013;4 1562.

67. Doorn KJ, et al. Microglial phenotypes and toll-like receptor 2 in the substantia nigra and hippocampus of incidental Lewy body disease cases and Parkinson's disease patients. Acta Neuropathol Commun. 2014;2:90

68. S. G. Daniele et al., Activation of MyD88-dependent TLR1/2 signaling by misfolded a-synuclein, a protein linked to neurodegenerative disorders. Sci Signal 8, ra45 (2015)

69. Zhang $W$, et al. Aggregated alpha-synuclein activates microglia: a process leading to disease progression in Parkinson's disease. FASEB J. 2005;19:533-42.

70. Fan Z, et al. Systemic activation of NLRP3 inflammasome and plasma asynuclein levels are correlated with motor severity and progression in Parkinson's disease. J Neuroinflammation. 2020;17:11.

71. Benner EJ, et al. Nitrated alpha-synuclein immunity accelerates degeneration of nigral dopaminergic neurons. PLoS One. 2008;3:e1376.

72. Reynolds $A D$, et al. Nitrated alpha-synuclein-activated microglial profiling for Parkinson's disease. J Neurochem. 2008;104:1504-25. 
73. Fellner $L$, et al. Toll-like receptor 4 is required for a-synuclein dependent activation of microglia and astroglia. Glia. 2013;61:349-60.

74. Li Y, et al. CXCL12 is involved in a-synuclein-triggered neuroinflammation of Parkinson's disease. J Neuroinflammation. 2019;16:263.

75. Kitada T, et al. Mutations in the parkin gene cause autosomal recessive juvenile parkinsonism. Nature. 1998;392:605-8.

76. Valente EM, et al. Hereditary early-onset Parkinson's disease caused by mutations in PINK1. Science. 2004;304:1158-60.

77. Koyano F, et al. Ubiquitin is phosphorylated by PINK1 to activate parkin. Nature. 2014;510:162-6.

78. Sliter DA, et al. Parkin and PINK1 mitigate STING-induced inflammation. Nature. 2018;561:258-62.

79. West AP, et al. Mitochondrial DNA stress primes the antiviral innate immune response. Nature. 2015;520:553-7.

80. Gui $C$, et al. p38 MAPK-DRP1 signaling is involved in mitochondrial dysfunction and cell death in mutant A53T a-synuclein model of Parkinson's disease. Toxicol Appl Pharmacol. 2019;388:114874.

81. Faustini $\mathrm{G}$, et al. Alpha-synuclein preserves mitochondrial fusion and function in neuronal cells. Oxid Med Cell Longev. 2019;2019:4246350.

82. Mitchell JD, Borasio GD. Amyotrophic lateral sclerosis. Lancet. 2007;369: 2031-41.

83. Kim HJ, Taylor JP. Lost in transportation: nucleocytoplasmic transport defects in ALS and other neurodegenerative diseases. Neuron. 2017;96:285-97.

84. Arai T, et al. TDP-43 is a component of ubiquitin-positive tau-negative inclusions in frontotemporal lobar degeneration and amyotrophic lateral sclerosis. Biochem Biophys Res Commun. 2006:351:602-11.

85. Neumann M, et al. Ubiquitinated TDP-43 in frontotemporal lobar degeneration and amyotrophic lateral sclerosis. Science. 2006;314:130-3.

86. Weskamp K, et al. Shortened TDP43 isoforms upregulated by neuronal hyperactivity drive TDP43 pathology in ALS. J Clin Invest. 2019.

87. Zhao W, et al. TDP-43 activates microglia through NF-kB and NLRP3 inflammasome. Exp Neurol. 2015;273:24-35.

88. Beers DR, Henkel JS, Zhao W, Wang J, Appel SH. CD4+ T cells support glial neuroprotection, slow disease progression, and modify glial morphology in an animal model of inherited ALS. Proc Natl Acad Sci U S A. 2008:105: 15558-63.

89. Boillée $\mathrm{S}$, et al. Onset and progression in inherited ALS determined by motor neurons and microglia. Science. 2006;312:1389-92.

90. Yamanaka $\mathrm{K}$, et al. Astrocytes as determinants of disease progression in inherited amyotrophic lateral sclerosis. Nat Neurosci. 2008;11:251-3.

91. Meissner F, Molawi K, Zychlinsky A. Mutant superoxide dismutase 1-induced IL-1 beta accelerates ALS pathogenesis. Proc Natl Acad Sci U S A. 2010;107: 13046-50.

92. Ayers JI, Fromholt SE, O'Neal VM, Diamond JH, Borchelt DR. Prion-like propagation of mutant SOD1 misfolding and motor neuron disease spread along neuroanatomical pathways. Acta Neuropathol. 2016;131:103-14.

93. Oono $\mathrm{M}$, et al. Transglutaminase 2 accelerates neuroinflammation in amyotrophic lateral sclerosis through interaction with misfolded superoxide dismutase 1. J Neurochem. 2014;128:403-18.

94. Maier M, et al. A human-derived antibody targets misfolded SOD1 and ameliorates motor symptoms in mouse models of amyotrophic lateral sclerosis. Sci Transl Med. 2018;10.

95. Kawamata T, Akiyama H, Yamada T, McGeer PL. Immunologic reactions in amyotrophic lateral sclerosis brain and spinal cord tissue. Am J Pathol. 1992; 140:691-707.

96. Rentzos M, et al. Interleukin-17 and interleukin-23 are elevated in serum and cerebrospinal fluid of patients with ALS: a reflection of Th17 cells activation? Acta Neurol Scand. 2010;122:425-9.

97. Saresella $\mathrm{M}$, et al. Thelper-17 activation dominates the immunologic milieu of both amyotrophic lateral sclerosis and progressive multiple sclerosis. Clin Immunol. 2013:148:79-88.

98. Chiu IM, et al. T lymphocytes potentiate endogenous neuroprotective inflammation in a mouse model of ALS. Proc Natl Acad Sci U S A. 2008;105: 17913-8.

99. Beers DR, et al. Endogenous regulatory T lymphocytes ameliorate amyotrophic lateral sclerosis in mice and correlate with disease progression in patients with amyotrophic lateral sclerosis. Brain. 2011;134:1293-314.

\section{Publisher's Note}

Springer Nature remains neutral with regard to jurisdictional claims in published maps and institutional affiliations.

Ready to submit your research? Choose BMC and benefit from:

- fast, convenient online submission

- thorough peer review by experienced researchers in your field

- rapid publication on acceptance

- support for research data, including large and complex data types

- gold Open Access which fosters wider collaboration and increased citations

- maximum visibility for your research: over $100 \mathrm{M}$ website views per year

At BMC, research is always in progress.

Learn more biomedcentral.com/submissions 